\title{
Applying how adults rehearse to understand how rehearsal may develop
}

\author{
Nelson Cowan* and Evie Vergauwe \\ Department of Psychological Sciences, University of Missouri, Columbia, MO, USA \\ *Correspondence: CowanN@missouri.edu
}

Edited by:

Christopher Jarrold, University of Bristol, UK

Reviewed by:

Alessandra S. Souza, University of Zürich, Switzerland

Jessica Spurgeon, University of Essex, UK

Keywords: development of rehearsal, verbal rehearsal, short-term memory, working memory, working memory development

We address short-term memory development and the role of covert verbal rehearsal (CVR), the imagined pronunciation of items from a list to be recalled. We suggest insights based on studies of serial and free recall. The research in adults has taken two tacks. (1) Overt rehearsal responses have been elicited to study what CVR strategies may occur, and (2) conditions have been imposed impeding the use CVR, presumably allowing an examination of its effects on recall. There are related lines of developmental research. We evaluate first overt rehearsal and then suppression-of-CVR evidence.

\section{OVERT REHEARSAL IN ADULTS}

After Rundus (1971), extensive follow-up research has been conducted (e.g., Tan and Ward, 2008; Bhatarah et al., 2009). Participants produced overt rehearsal between item presentations however they wished, preceding serial or free recall (precued or post-cued). Similar patterns of rehearsal were found in all four situations. Tan and Ward (2008) suggested that "When participants rehearsed, they tended to do so in a cumulative forward order up to Serial Position 4, after which the amount of rehearsal decreased substantially." After Position 4, assuming that time to rehearse was not the issue, rehearsal could have decreased because of a capacity limit in how many items can be remembered concurrently for rehearsal (Cowan, 2001). Participants appear to want to rehearse from the first item onward and don't find it worthwhile to rehearse subsequences starting later in the list.

One caution, though, is that it is theoretically possible for CVR to occur with words overlapping in time, which is impossible for overt rehearsal. Moreover, findings of Bhatarah et al. (2009) suggest that Tan and Ward's (2008) description is incomplete. Figure 3 of Bhatarah et al. shows the number of words from each input serial position that were overtly rehearsed after Input 1, after Input 2, and so on through Input 8 . This figure does show that the frequency of rehearsals decreases as a function of the input position. It also shows, though, a recent effect in rehearsal after each input position, extending to the last three or four items. That pattern could not result from rehearsal always starting at the beginning of the list. Instead, it suggests that sometimes there is rehearsal of recent subsequences of a few items.

This finding of non-initial subsequence rehearsal, not emphasized by Bhatarah et al. (2009), potentially has implications regarding the purpose of CVR. According to Baddeley (1986), the purpose is to reactivate phonological representations before they decay to the point of becoming unavailable for recall. For that purpose, it would make sense to reactivate the least recently encountered or rehearsed items, not the few most recently encountered and not consistently the list from its beginning. Other purposes of rehearsal are possible, however.

Rehearsal could result in a stronger chaining of associations between consecutive words in long-term memory. In that case, one might observe a moving window of rehearsal, e.g., rehearsal of Words 3-4-5, 4-5-6, 5-6-7, etc. We know that chaining cannot be the sole mechanism of recall, given considerable evidence in serial recall against it, favoring item-to-serial-position associations (e.g., Lee and Estes, 1981; Henson et al., 1996). Still, the participant might rehearse a list-medial sequence while thinking about where that sequence is to go in the list, perhaps starting where the previous rehearsal cycle left off.

Another possible purpose of rehearsal is to support grouping, the beginning of the formation of a new chunk in memory (cf., Anderson and Matessa, 1997; Cowan, 2001; Farrell, 2012). If so, a moving window of rehearsal would not be expected. A close analysis might reveal, instead, that participants often rehearse Items 1-2-3 several times and then start rehearsing Items 4-5-6, forming two separate groups, etc. We anticipate in any case that the pattern shown in rehearsal, when viewed in greater detail, will be reproduced in free recall (Laming, 2006).

An objection to the idea of rehearsal in serial recall is that there is not enough time to carry out rehearsal of the list. If the rehearsal following each item does not have to begin at the start of the list, though, there could be enough time, and it could strengthen item-to-item and/or item-toposition associations.

\section{DEVELOPMENT OF OVERT REHEARSAL}

Flavell et al. (1966) exploited the notion that action patterns start out overt and later are internalized, to document the increase in rehearsal for serial recall during childhood. The experimenter pointed to named pictures at a rate of $2 \mathrm{~s}$ per picture. In delayed recall, there was a 15-s maintenance period before the child tried to point to the pictures in the presented order. To make children less self-conscious during the retention interval, they wore a space helmet with a translucent visor that came 
down during the interval but did not cover the mouth. An experimenter trained in lip-reading could see and hear overt verbal rehearsal, mostly in the form of soft whispering or soundless mouth movements. These steadily increased in number from kindergarten $(\sim 5$ years $)$ to second grade ( $\sim 7$ years) to fifth grade $(\sim 10$ years $)$, suggesting that rehearsal increases markedly during these elementary school years.

There has been more such work in free recall, providing greater specificity to what is rehearsed. Ornstein and Naus (1978) summarized work showing that, with maturation during the elementary school years, overt rehearsal becomes more cumulative. Guttentag et al. (1987) provided more specificity. They asked children from third through sixth grades $(\sim 8$ to $\sim 11$ years) to think aloud while receiving audiovisual presentations of words for free recall. Overt rehearsal progressed from sets of 1.4 words in third grade to about 4.2 and 4.5 words in fourth and sixth grades. The constraint on rehearsal set size was apparently working memory capacity; all age groups increased the rehearsal set size considerably when each item remained visually available after its presentation (to 2.9, 7.0, and 7.4 in the three age groups). This work has continued with Lehmann and Hasselhorn (2007, 2010, 2012). The 2007 work confirmed directly that capacity limits do restrict the use of cumulative rehearsal, and the later work showed that children's rehearsal patterns conform to adult findings. Sequences in overt rehearsal tended to show up in recall, as in the adult work of Laming (2006), and rehearsal sequences tended toward forward item-to-item associations as in adults (Kahana, 1996). Thus, we suspect that CVR occurs in both serial and free recall and that rehearsal set size increases with age. Rehearsal of several items in a burst often, but not always, begins at list Position 1 .

In one study (Cowan et al., 2006a), second-grade children and adults were asked how they carried out a digit span task. Most of the adults vs. none of the second-graders said that they grouped the items, suggesting that there may well be dramatic change in rehearsal style with development.

The natural observation of rehearsal cannot establish the causal role of rehearsal in recall. A positive intervention might, but an attempt to increase the speed of rehearsal to help recall was unsuccessful; the speed of rehearsal could not be increased (Hulme and Muir, 1985). In principle, a causal role of rehearsal might be established by impeding rehearsal and examining the effect on recall, as we now discuss.

\section{IMPEDING REHEARSAL IN ADULTS}

Short-term memory capacity is diminished when rehearsal is impeded (Baddeley, 1986). To establish a causal connection between CVR and recall, one must take certain precautions. One must ensure that the effect of impeding rehearsal is not due to the interference effects of the rehearsal-suppression task. These interference effects occur when the rehearsal-suppression stimulus changes over time (Oberauer and Lewandowsky, 2008). One also must ensure that the stimulus is encoded phonologically, making it suitable for CVR; suppressing rehearsal impedes phonological encoding for printed but not for spoken stimuli (Baddeley, 1986).

One expectation is that, if CVR is causal, suppression of rehearsal will reduce or eliminate effects attributed to CVR, including detrimental effects of phonological similarity and word length. Cowan et al. (1987) presented lists of words for serial recall that were phonologically similar (bat, hat, etc.) or dissimilar, through headphones to ensure phonological encoding, and sometimes required various kinds of rehearsal suppression in a whispered voice during the presentation. The suppression task changed during some trials (e.g., successive letters of the alphabet to be whispered after each list item) and sometimes remained the same (the same letter to be whispered after all list items). Word span was reduced the most overall when the suppression letters changed during the trial, as Oberauer and Lewandowsky (2008) would expect. Even when the suppression item did not change, though, the phonological similarity effect was diminished almost as much as when the suppression items did change within a trial. Thus, beyond interference effects of changing suppression stimuli, we believe that suppression from a repeated stimulus hinders recall by preventing phonological rehearsal during presentation of the list.

Instead of relying on simple quantitative effects of suppressing rehearsal, one can set up tasks allowing alternative strategies that either include or exclude CVR. Cowan et al. (2006b) set up a task in which names appeared one at a time at different house locations. The participant received a name probe and was to drag it to the house in which it had appeared. Sometimes the names had to appear one to a house, and a simple strategy was to remember separately the path between houses and the serial sequence of names. For example, the third name in the remembered list must have appeared in the third house in the remembered path. On other trials, some houses remained vacant but multiple names appeared at other houses (not in immediate succession), so that the path between houses became convoluted. Then, remembering the serial sequences of houses was difficult and the participant had to rely on direct house-name associations. The results showed that adults tended to use the path- and word-sequencing method (doing better when the paths were not convoluted) unless rehearsal suppression was required, in which case they switched to the house-name association method (doing better when the paths were convoluted because fewer houses had names). It was suggested that the path and sequencerehearsal method required less attention.

\section{IMPEDING REHEARSAL: A COMPARISON WITH CHILDREN}

One might attempt to impede CVR in children. If young children do not rehearse effectively, impeding rehearsal should have little effect on their recall. In practice, this research strategy is problematic because rehearsal is more attention-demanding in young children than it is in adults (Guttentag, 1984).

Another research strategy is to ask whether suppression of rehearsal in adults makes them perform like children. Cowan et al. (1987) did find that rehearsal suppression made adults resemble 6- to 8-year-old children in their level of recall performance and magnitude of the phonological similarity effect; caution is needed in interpreting age changes in the magnitude of the phonological similarity 
effect, however (Jarrold and Citroën, 2013). Cowan et al. (2006b) found that third-grade children showed a pattern of recall suggesting a direct word-house association method that did not require CVR, much like adults under rehearsal suppression.

\section{CONCLUSION}

In traditional approaches toward rehearsal, it is needed to reactivate items in a repeating loop before they decay. There are several reasons to suspect that this function of rehearsal is discrepant from the overt rehearsal protocols and recall records, and therefore is not suitable to explain the development of rehearsal. We have suggested some alternative schedules and functions of rehearsal (a moving window to strengthen associations, or separate item groups), and have presented evidence suggesting that rehearsal increases with development in a manner that might assist both serial and free recall. Capacity limits may restrict young children's ability to use rehearsal, in which case rehearsal can be said to amplify the benefits of the growth of capacity. It is possible that gradual, quantitative changes in rehearsal ability have abrupt, qualitative, step-like consequences on the development of recall because a certain level of ability may be needed to make CVR actually useful in a given recall task.

\section{ACKNOWLEDGMENTS}

Funding for this project was provided by NIH Grant R01 HD-21338 to Nelson Cowan and by Swiss National Science Foundation Grant \# PA00P1_139604 to Evie Vergauwe.

\section{REFERENCES}

Anderson, J. R., and Matessa, M. (1997). A production system theory of serial memory. Psychol. Rev. 104, 728-748.
Baddeley, A. D. (1986). Working Memory. Oxford, England: Clarendon Press.

Bhatarah, P., Ward, G., Smith, J., and Hayes, L. (2009). Examining the relationship between free recall and immediate serial recall: similar patterns of rehearsal and similar effects of word length, presentation rate and articulatory suppression. Mem. Cognit. 37, 689-713. doi: 10.3758/MC.37.5.689

Cowan, N. (2001). The magical number 4 in shortterm memory: a reconsideration of mental storage capacity. Behav. Brain Sci. 24, 87-185. doi: 10.1017/S0140525X01003922

Cowan, N., Cartwright, C., Winterowd, C., and Sherk, M. (1987). An adult model of preschool children's speech memory. Mem. Cognit. 15, 511-517.

Cowan, N., Elliott, E. M., Saults, J. S., Nugent, L. D., Bomb, P., and Hismjatullina, A. (2006a). Rethinking speed theories of cognitive development: increasing the rate of recall without affecting accuracy. Psychol. Sci. 17, 67-73. doi: 10.1111/j.1467-9280.2005.01666.x

Cowan, N., Saults, J. S., and Morey, C. C. (2006b). Development of working memory for verbalspatial associations. J. Mem. Lang. 55, 274-289. doi: $10.1016 /$ j.jml.2006.04.002

Farrell, S. (2012). Temporal clustering and sequencing in short-term memory and episodic memory. Psychol. Rev. 119, 223-271. doi: 10.1037/a0027371

Flavell, J. H., Beach, D. H., and Chinsky, J. M. (1966). Spontaneous verbal rehearsal in a memory task as a function of age. Child Dev. 37, 283-299.

Guttentag, R. E. (1984). The mental effort requirement of cumulative rehearsal: a developmental study. J. Exp. Child Psychol. 37, 92-106.

Guttentag, R. E., Ornstein, P. A., and Siemens, L. (1987). Children's spontaneous rehearsal: transitions in strategy acquisition. Cogn. Dev. 2, 307-326.

Henson, R. N. A., Norris, D. G., Page, M. P. A., and Baddeley, A. D. (1996). Unchained memory: error patterns rule out chaining models of immediate serial recall. Q. J. Exp. Psychol. A 49, 80-115.

Hulme, C., and Muir, C. (1985). Developmental changes in speech rate and memory span: a causal relationship? Br. J. Dev. Psychol. 3, 175-181.

Jarrold, C., and Citroën, R. (2013). Reevaluating key evidence for the development of rehearsal: phonological similarity effects in children are subject to proportional scaling artifacts. Dev. Psychol. 49, 837-847. doi: 10.1037/a0028771

Kahana, M. J. (1996). Associative retrieval processes in free recall. Mem. Cognit. 24, 103-109.

Laming, D., (2006). Predicting free recalls. J. Exp. Psychol. Learn. Mem. Cogn. 32, 1146-1163. doi: 10.1037/0278-7393.32.5.1146
Lee, C. L., and Estes, W. K. (1981). Item and order information in short term memory: evidence for multilevel perturbation processes. J. Exp. Psychol. Hum. Learn. Mem. 7, 149-169.

Lehmann, M., and Hasselhorn, M. (2007). Variable memory strategy use in children's adaptive intratask learning behavior: developmental changes and working memory influences in free recall. Child Dev. 78, 1068-1082. doi: 10.1111/j.1467-8624.2007.01053.x

Lehmann, M., and Hasselhorn, M. (2010) The dynamics of free recall and their relation to rehearsal between 8 and 10 years of age. Child Dev. 81, 1006-1020. doi: 10.1111/j.1467-8624.2010.01448.x

Lehmann, M., and Hasselhorn, M. (2012). Rehearsal dynamics in elementary school children. J. Exp. Child Psychol. 111, 552-560. doi: 10.1016/j.jecp.2011.10.013

Oberauer, K., and Lewandowsky, S. (2008). Forgetting in immediate serial recall: decay, temporal distinctiveness, or interference? Psychol. Rev. 115, 544576. doi: 10.1037/0033-295X.115.3.544

Ornstein, P. A., and Naus, M. J. (1978). "Rehearsal processes in children's memory," in Memory Development in Children, ed P. A. Ornstein (Hillsdale, NJ: Erlbaum), 69-99.

Rundus, D. (1971). Analysis of rehearsal processes in free recall. J. Exp. Psychol. 89, 63-77.

Tan, L., and Ward, G. (2008). Rehearsal in immediate serial recall. Psychon. Bull. Rev. 15, 535-542. doi: 10.3758/PBR.15.3.535

Conflict of Interest Statement: The authors declare that the research was conducted in the absence of any commercial or financial relationships that could be construed as a potential conflict of interest.

Received: 08 October 2014; accepted: 12 December 2014; published online: 07 January 2015.

Citation: Cowan $N$ and Vergauwe E (2015) Applying how adults rehearse to understand how rehearsal may develop. Front. Psychol. 5:1538. doi: 10.3389/fpsyg. 2014.01538

This article was submitted to Developmental Psychology, a section of the journal Frontiers in Psychology.

Copyright (c) 2015 Cowan and Vergauwe. This is an open-access article distributed under the terms of the Creative Commons Attribution License (CC BY). The use, distribution or reproduction in other forums is permitted, provided the original author(s) or licensor are credited and that the original publication in this journal is cited, in accordance with accepted academic practice. No use, distribution or reproduction is permitted which does not comply with these terms. 